\title{
Age at First Episode
}

National Cancer Institute

\section{Source}

National Cancer Institute. Age at First Episode. NCI Thesaurus. Code C124438.

The age at which the first episode occurred. 